\title{
Axonal Reconstructions Going Live
}

\author{
Alison J. Canty • Vincenzo De Paola
}

Published online: 19 April 2011

(C) The Author(s) 2011. This article is published with open access at Springerlink.com

Keywords Axons · Cortex · In vivo 2-photon imaging · Synapses $\cdot$ Synaptic plasticity $\cdot$ Sensory experience . Connectivity $\cdot$ Neuroanatomy $\cdot$ DIADEM

The cellular and circuit mechanisms of experiencedependent plasticity and repair in the mammalian neocortex are the subject of intensive study. Axonal networks and their synapses are of particular interest as they play a key role in numerous neurological diseases including injury and neurodegeneration ${ }^{1,2}$. Tracking axons in living animals over a variety of temporal and spatial scales has recently become possible, using a combination of genetically encoded fluorophores, 2-photon (2P) microscopy and neuroanatomy ${ }^{3}$. This has opened the possibility of designing more powerful experiments to directly test the involvement of axonal structural remodeling in a variety of physiological processes, including learning and regeneration. We argue here that substantial advancement in our knowledge of axonal plasticity will rely on the development of new

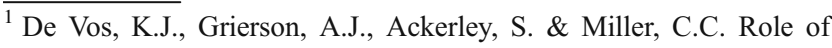
axonal transport in neurodegenerative diseases. Annu Rev Neurosci 31, 151-173 (2008).

${ }^{2}$ Cafferty, W.B., McGee, A.W. \& Strittmatter, S.M. Axonal growth therapeutics: regeneration or sprouting or plasticity? Trends Neurosci 31, 215-220 (2008).

${ }^{3}$ De Paola, V., et al. Cell type-specific structural plasticity of axonal branches and boutons in the adult neocortex. Neuron 49, 861-875 (2006).

A. J. Canty $\cdot$ V. De Paola $(\varangle)$

MRC Clinical Sciences Centre, Imperial College London,

Du cane rd,

W120NN, London, UK

e-mail: Vincenzo.depaola@imperial.ac.uk

Present Address:

A. J. Canty

School of Medicine, University of Tasmania,

Private Bag 34,

Hobart 7000, Australia
}

analytical tools that can automate the quantitative reconstruction of these fine processes.

The mammalian cortex is comprised of 6 layers. Axons form a complex network throughout all these layers. For example in layer 1 (L 1) axonal projections originate locally, contra-laterally and sub-cortically extending over several millimeters and contacting thousands of neurons on the way, making it an area of diverse inputs. This is particularly evident in the somatosensory cortex, where the primary function is to integrate and process incoming information from multiple sensory receptors, but is expected to be true for other cortical areas as well.

Mapping the trajectories of neurons in the cortex has traditionally been performed by single cell labeling or Golgi impregnation methods. Thick cortical blocks are then analyzed using manual tracing software such as Neurolucida (or similar) to create 3 dimensional (3D) reconstructions of neurons and their processes. This is a laborious and time consuming task not only in generating labeled tissue of a handful of neurons per preparation, but especially in the manual tracing and reconstruction of the neuronal projections $^{4}$. For example, the axon of a single L $2 / 3$ pyramidal cell in barrel cortex can extend over $60 \mathrm{~mm}$ in length ${ }^{5}$. Building a map of the projections for each neuron in the brain is both a huge challenge and of exceptional value. As neuroscientist Ramon y Cajal remarkably realized over 100 years ago, by staring at fixed tissue samples, axonal morphology delineates the path of information flow within the brain. Studying the details of axonal connectivity and especially of its dynamics will likely continue to provide insights into the principles of brain organization and neuronal communication in the future.

\footnotetext{
${ }^{4}$ Brown, K.M., et al. The DIADEM Data Sets: Representative Light Microscopy Images of Neuronal Morphology to Advance Automation of Digital Reconstructions. Neuroinformatics (2011).

${ }^{5}$ Bruno, R.M., Hahn, T.T., Wallace, D.J., de Kock, C.P. \& Sakmann, B. Sensory experience alters specific branches of individual corticocortical axons during development. J Neurosci 29, 3172-3181 (2009).
} 
In recent years two technological advancements in imaging neuronal structure have enormously facilitated this task. 1) By expressing green fluorescent protein (GFP) under the control of the Thy 1 promoter $^{6}$ in the mouse, a sub-population of projection neurons can be visualized in situ in the brain. Targeting GFP to either the cytosol (GFP-M line) $)^{7}$ or the membrane (Line 15$)^{8}$ results in labeling of the entire neuronal arbor including not only terminal branches but also synapses on both axons and dendrites (i.e. boutons and spines, respectively), making it possible to reconstruct sub-components of the cortical circuitry in the whole mount brain. 2) $2 \mathrm{P}$ microscopy ${ }^{9}$ has revolutionized the way we study neuronal plasticity. By combining $2 \mathrm{P}$ imaging with either a thinned skull or cranial window preparation, it is now possible to directly visualize cortical networks over a wide range of temporal scales, several hundred micrometers deep in the living brain (i.e. typically over L 1-3) (refer to ${ }^{10,11,12}$ for further discussion on the advantages and disadvantages of each preparation). These experiments are beginning to reveal that although the overall morphology of dendrites and the majority of synaptic structures are remarkably stable in the mature brain, experience-dependent plasticity, learning and neuronal damage are accompanied by a degree of structural remodeling of spines ${ }^{13,14,15}$.

As cortical axons are thinner in diameter, longer and follow more tortuous paths than dendrites, they are more difficult to track in vivo and much less is known about axonal organization and dynamics ${ }^{16}$. In vivo $2 \mathrm{P}$ microscopy is an ideal approach to tackle this task for two reasons: 1 )

\footnotetext{
${ }^{6}$ Caroni, P. Overexpression of growth-associated proteins in the neurons of adult transgenic mice. J Neurosci Methods 71, 3-9 (1997).

${ }^{7}$ Feng, G., et al. Imaging neuronal subsets in transgenic mice expressing multiple spectral variants of GFP. Neuron 28, 41-51 (2000).

${ }^{8}$ De Paola, V., Arber, S. \& Caroni, P. AMPA receptors regulate dynamic equilibrium of presynaptic terminals in mature hippocampal networks. Nat Neurosci 6, 491-500. (2003).

${ }^{9}$ Svoboda, K. \& Yasuda, R. Principles of two-photon excitation microscopy and its applications to neuroscience. Neuron 50, 823-839 (2006).

${ }^{10}$ Yang, G., Pan, F., Parkhurst, C.N., Grutzendler, J. \& Gan, W.B. Thinned-skull cranial window technique for long-term imaging of the cortex in live mice. Nat Protoc 5, 201-208 (2010).

${ }^{11}$ Holtmaat, A., et al. Long-term, high-resolution imaging in the mouse neocortex through a chronic cranial window. Nat Protoc 4, 1128-1144 (2009).

${ }^{12}$ Xu, H.T., Pan, F., Yang, G. \& Gan, W.B. Choice of cranial window type for in vivo imaging affects dendritic spine turnover in the cortex. Nat Neurosci 10, 549-551 (2007).

${ }^{13} \mathrm{Yu}, \mathrm{X}$. \& Zuo, Y. Spine plasticity in the motor cortex. Curr Opin Neurobiol (2010)

${ }^{14}$ Murphy, T.H. \& Corbett, D. Plasticity during stroke recovery: from synapse to behaviour. Nat Rev Neurosci 10, 861-872 (2009).

${ }^{15}$ Holtmaat, A. \& Svoboda, K. Experience-dependent structural synaptic plasticity in the mammalian brain. Nat Rev Neurosci 10, 647-658 (2009).

${ }^{16}$ Gogolla, N., Galimberti, I. \& Caroni, P. Structural plasticity of axon terminals in the adult. Curr Opin Neurobiol 17, 516-524 (2007).
}

Dense networks of horizontally projecting axons can be visualized in superficial cortical layers. For example L 1 is primarily devoid of cell bodies but richly packed in axonal and dendritic processes. 2) Longitudinal live imaging studies of single axons can provide mechanistic insights into the principles of branch and synaptic remodeling ${ }^{3}$, which are going to be difficult to deduce from analysis of fixed preparations. Some progress has recently been made in identifying the origin of the axons imaged in vivo. This is based on their branching patterns and synaptic makeup in the upper layers of the cortex. Post-mortem histological analysis and serial-section reconstruction of the entire axonal arbors imaged with $2 \mathrm{P}$ microscopy led to the identification of subpopulations of neurons residing within L 2/3, 5 and 6 and thalamus ${ }^{3}$. Vertical projections are more difficult to classify with $2 \mathrm{P}$ microscopy given the limited $\mathrm{z}$ resolution and their plasticity is best-studied using single cell labeling and histological reconstructions ${ }^{5,17,18}$.

One would like to understand the complexity of axonal networks in the cortex, but also their ability to adapt in the case of experience-dependent plasticity, learning or neurological damage. For example, manipulation of the whiskers is likely to result in direct changes in connectivity that can be mapped in the corresponding barrel fields of the sensory cortex $^{19,20}$ (Song et al, in preparation). Furthermore, lesion studies either of the whiskers, or more directly induced in the cortex by blood vessel occlusion or laser-mediated ablation are likely to result in axonal synaptic structural rearrangements (Canty et al., in preparation). The current challenge we are facing is to resolve and to reliably track structural changes over various spatial scales, ranging from a few micrometers, i.e. the scale of synaptic gain and loss or size changes, to fractions of a millimeter, i.e. the scale of axonal branch extension and retraction, in a variety of experimental settings (sensory stimulation, deprivation, learning, traumatic injury, etc.) and over different time intervals and developmental ages ${ }^{21,22,23}$.

\footnotetext{
${ }^{17}$ Broser, P., Grinevich, V., Osten, P., Sakmann, B. \& Wallace, D.J. Critical period plasticity of axonal arbors of layer $2 / 3$ pyramidal neurons in rat somatosensory cortex: layer-specific reduction of projections into deprived cortical columns. Cereb Cortex 18, 15881603 (2008).

${ }^{18}$ Wimmer, V.C., Broser, P.J., Kuner, T. \& Bruno, R.M. Experienceinduced plasticity of thalamocortical axons in both juveniles and adults. J Comp Neurol 518, 4629-4648 (2010).

${ }^{19}$ Feldman, D.E. \& Brecht, M. Map plasticity in somatosensory cortex. Science 310, 810-815 (2005).

${ }^{20}$ Barnes, S.J. \& Finnerty, G.T. Sensory experience and cortical rewiring. Neuroscientist 16, 186-198 (2010).

${ }^{21}$ Chklovskii, D.B., Mel, B.W. \& Svoboda, K. Cortical rewiring and information storage. Nature 431, 782-788 (2004).

${ }^{22}$ Hubener, M. \& Bonhoeffer, T. Searching for engrams. Neuron 67, 363-371 (2010)

${ }^{23}$ Butz, M., Worgotter, F. \& van Ooyen, A. Activity-dependent structural plasticity. Brain Res Rev 60, 287-305 (2009).
} 
New automated methods need to be developed to assure a reproducible analysis and avoid bias due to different users $^{24,25,26}$. Ideally, one would like to be able to sample large $(\mathrm{mm})$ volumes of cortical tissue over time. This is necessary to map structural changes in both basal conditions and associated with a given sensory experience or damage in several hundreds of neurons at the same time. In particular, axonal reconstructions from time lapses acquired in vivo will pose unique computational difficulties in terms of variable image quality, movement artifact and alignment across imaging sessions. As a first step in this process, accurate semi-automated reconstruction software in $3 \mathrm{D}$, as the ones developed for the DIADEM challenge (www. diademchallenge.org) ${ }^{27,28,29,30,31,32}$, could facilitate such direct and high-throughput comparison of axonal projections over time and the mapping of large-scale changes in the cortex of living animals.

Where do we go from here? To further our understanding of axonal reorganization a more complete and systematic characterization of the trajectory and activity of genetically determined subtypes of L 1-3 projecting neurons would be desirable ${ }^{33}$. For example, this can be achieved by combining new Cre-expressing lines with strong XFP reporters and cell type-specific promoters ${ }^{34,35}$.

The in vivo data sets supplied for the DIADEM challenge were entirely axonal, because there is an

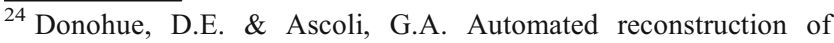
neuronal morphology: An overview. Brain Res Rev.

${ }^{25}$ Broser, P.J., et al. Automated axon length quantification for populations of labelled neurons. J Neurosci Methods 169, 43-54 (2008).

${ }^{26}$ Oberlaender, M., Bruno, R.M., Sakmann, B. \& Broser, P.J.

Transmitted light brightfield mosaic microscopy for threedimensional tracing of single neuron morphology. J Biomed Opt 12, 064029 (2007).

${ }^{27}$ Bas E, E.D. Principal Curves as Skeletons of Tubular Objects: Locally Characterizing the Structures of Axons. Neuroinformatics (2011).

${ }^{28}$ Chothani P, M.V., Stepanyants A Automated tracing of neurites from light microscopy stacks of images. Neuroinformatics (2011).

${ }^{29}$ Turetken E, G.G., Blum C, Fua P Automated Reconstruction of > Dendritic and Axonal Trees by Global Optimization with Geometric Priors. Neuroinformatics (2011).

${ }^{30}$ Zhao T, X.J., Amat F, Clack N, Ahammad P, Peng H, Long F, Myers E Automated Reconstruction of Neuronal Morphology Based on Local Geometrical and Global Structural Models. (2011).

${ }^{31}$ Wang Y, N.A., Tsai C, Roysam B Generally Applicable 3D > Neuron Tracing Approach and System based on Open-Curve Snake. (2011).

${ }^{32}$ Narayanaswamy A, W.Y., Roysam B A,. Preprocessing Pipeline to Enhance 3-D Images of Neuronal Arbors. . Neuroinformatics (2011).

${ }^{33}$ Luo, L., Callaway, E.M. \& Svoboda, K. Genetic dissection of neural circuits. Neuron 57, 634-660 (2008).

${ }^{34}$ Madisen, L., et al. A robust and high-throughput Cre reporting and characterization system for the whole mouse brain. Nat Neurosci 13, 133-140 (2009).

${ }^{35}$ Novak, A., Guo, C., Yang, W., Nagy, A. \& Lobe, C.G. Z/EG, a double reporter mouse line that expresses enhanced green fluorescent protein upon Cre-mediated excision. Genesis 28, 147-155 (2000).
}

urgent need to advance our knowledge of axonal biology in particular regarding the contribution of axonal dynamics to cortical plasticity and repair ${ }^{19}$. However, we would anticipate extending the automated algorithms to include dendritic arbors and the alignment of post-mortem tangential brain sections. This will allow to trace the origin of projections to underlying cell bodies in the deeper layers of the cortex or even sub cortically, leading to the reconstruction of full axonal and dendritic trees and not just their L 1-3 portions. Recent advances in the highthroughput generation of electron microscopy data ${ }^{36,37,38}$ hold great promise in adding ultra structural and circuitlevel information to the axonal reconstructions from live imaging experiments. Finally, automated tools to track not only neuronal arbors but also synaptic sites, synaptic proteins $^{39,40,41,42}$, organelles such as mitochondria ${ }^{43}$, and synaptic activity ${ }^{44,45,46}$ in living cortical axons will enormously speed up the analysis of axonal plasticity and shed light on the physiological settings that regulate neuronal structure and function in the brain.

Open Access This article is distributed under the terms of the Creative Commons Attribution Noncommercial License which permits any noncommercial use, distribution, and reproduction in any medium, provided the original author(s) and source are credited.

\footnotetext{
${ }^{36}$ Knott, G., Marchman, H., Wall, D. \& Lich, B. Serial section scanning electron microscopy of adult brain tissue using focused ion beam milling. J Neurosci 28, 2959-2964 (2008).

${ }^{37}$ Helmstaedter, M., Briggman, K.L. \& Denk, W. 3D structural imaging of the brain with photons and electrons. Curr Opin Neurobiol 18, 633-641 (2008).

${ }^{38} \mathrm{Li}$, J., Erisir, A. \& Cline, H. In vivo time-lapse imaging and serial section electron microscopy reveal developmental synaptic rearrangements. Neuron 69, 273-286 (2011).

${ }^{39}$ Gray, N.W., Weimer, R.M., Bureau, I. \& Svoboda, K. Rapid redistribution of synaptic PSD-95 in the neocortex in vivo. PLoS Biol 4, e370 (2006).

${ }^{40}$ Minerbi, A., et al. Long-term relationships between synaptic tenacity, synaptic remodeling, and network activity. PLoS Biol 7, e1000136 (2009).

${ }^{41}$ Ruthazer, E.S., Li, J. \& Cline, H.T. Stabilization of axon branch dynamics by synaptic maturation. $J$ Neurosci 26, 3594-3603 (2006).

${ }^{42}$ Meyer, M.P. \& Smith, S.J. Evidence from in vivo imaging that synaptogenesis guides the growth and branching of axonal arbors by two distinct mechanisms. J Neurosci 26, 3604-3614 (2006).

${ }^{43}$ Misgeld, T., Kerschensteiner, M., Bareyre, F.M., Burgess, R.W. \& Lichtman, J.W. Imaging axonal transport of mitochondria in vivo. Nat Methods 4, 559-561 (2007).

${ }^{44}$ Dreosti, E., Odermatt, B., Dorostkar, M.M. \& Lagnado, L. A genetically encoded reporter of synaptic activity in vivo. Nat Methods 6, 883-889 (2009).

${ }^{45}$ Miesenbock, G. The optogenetic catechism. Science 326, 395-399 (2009).

${ }^{46}$ Mank, M., et al. A FRET-based calcium biosensor with fast signal kinetics and high fluorescence change. Biophys $J$ 90, 1790-1796 (2006).
} 